\title{
Signal-to-Noise ratio and design complexity based on Unified Loss Function - LTB case with Finite Target
}

\author{
N.K. Sharma ${ }^{1}$, E.A. Cudney ${ }^{1}$ * \\ ${ }^{1 *}$ Department of Engineering Management and Systems Engineering, Missouri University of Science and Technology Rolla, MO, USA \\ *Corresponding Author: e-mail: cudney@mst.edu, Tel 573-3417931, Fax. 573-3416567
}

\begin{abstract}
Taguchi's quality loss function for larger-the-better performance characteristics uses a reciprocal transformation to compute quality loss. This paper suggests that reciprocal transformation unnecessarily complicates and may distort results. Examples of this distortion include the signal-to-noise ratio based on mean squared deviation and the signal-to-noise ratio based on complexity. The concept of complexity is an important element of axiomatic design and axiomatic quality. This paper shows that a simple linear transformation as used in the unified loss function can give an appropriate and comparable signal-to-noise ratio based on mean squared deviation and signal-to-noise ratio based on complexity for larger-the-better characteristics. Mathematical derivations are given and two examples are discussed to demonstrate the proposed methodology.
\end{abstract}

Keywords: Design Complexity, Axiomatic Design, Signal-to-Noise Ratio, Robust Design, Quality Loss Function.

DOI: http://dx.doi.org/10.4314/ijest.v3i7.2S

\section{Introduction}

Two types of performance characteristics have been discussed in the literature on quality engineering (Fowlkes and Creveling, 1995; Taguchi et al, 2004; Taguchi et al, 1999). First, the characteristics, which have the maximum possible target as $100 \%$, are not the larger-the-better characteristics (Taguchi et al, 2004). Second, the characteristics, which have infinity as the target value, do not really have a target value and are LTB characteristics. In other words, a performance characteristic should have a target as infinity for it to be designated as an LTB characteristic, and characteristics that have a limitation in the theoretical target value cannot be designated as LTB.

A unified quality loss function was proposed in Sharma et al (2007) to bring about similarity among the three cases including smaller-the-better (STB), nominal-the-best (NTB), and larger-the-better (LTB). They proposed a unified formula for quality loss by introducing a term called the target-mean ratio. Implications of the unified quality loss function on the field of quality engineering were studied in Sharma et al (2008). This paper attempts to further study the implications of the unified quality loss function on the SN ratio, the concept of complexity in axiomatic design, and the relationship between complexity and SN ratio. It will be shown that the complexity formulation for LTB with an infinite target is not equivalent to that for STB and NTB characteristics. Therefore, complexity due to variability for LTB with a finite target is derived, and it is found that a comparable complexity measure is obtained using the unified quality loss function. The effect of a finite target for LTB on the mathematical relationship between the SN ratio and axiomatic measures has also been studied. It will be also shown that for LTB the relationship between the $\mathrm{SN}$ ratio and complexity becomes equivalent to the STB case.

There are two important axioms in axiomatic design. The independence axiom of axiomatic design is "maintain the independence of the functional requirements" and the information axiom of axiomatic design is "minimize the information content of the design" (Suh, 2001). This paper considers in detail the information axiom because complexity is measured using information content. This paper is not explicitly concerned with the independence axiom because a single functional requirement or quality characteristic is considered.

Axiomatic design research has advanced the design practices toward more useful arenas. One of these arenas is related to the 
elimination or minimization of vulnerabilities that are established in the design entity due to the violation of design axioms (ElHaik, 2005). Design complexity, which is addressed by the information axiom, is a major vulnerability. Complexity has three components including sensitivity, variability, and correlation. The information measure has been used to derive mathematical relationships that quantify these components in the context of the axiomatic quality process (El-Haik, 2005).

The robust design philosophy proposed focuses upstream on the conceptual design stage. In contrast, traditional approaches normally focus downstream. An important objective in robust design is to reduce the sensitivity of system performance to variation due to manufacturing, conditions of customer use, and wear. Axiomatic design and Taguchi methods have been linked via information content in design (Kar, 2000). Kar (2000) showed that the information content in product and process design is directly proportional to Taguchi's quality loss function and inversely proportional to the SN ratio. The paper established a rigorous link between axiomatic design methods and robust design methods using information content to indicate that a product, which has low information content, has low quality loss and high SN ratio. Kar used Shannon's communication channel and entropy definition from information theory and Kullback's symmetric divergence measure for information content to establish links between both the design arenas. However, for LTB the reciprocal transformation was used.

El-Haik contributed to bridging the gap between axiomatic design and robust design through mathematical relationships. The integration of the axiomatic design method with robust design provides useful perspectives of the design (El-Haik, 2006). This contribution involves two parts, which include (1) complexity related to quality loss functions and (2) complexity related to SN ratios for STB, NTB, and LTB characteristics. Quality loss is a function of variance and bias (i.e., mean deviation from the target) and complexity is a function of variance. El-Haik (2005) related complexity reduction with reduction in quality loss. However, the reduction in complexity is different based on the type of characteristic; e.g., STB and LTB. This is because of the reciprocal transformation that has been used for LTB, whereas STB (and NTB) use linear transformation. This research argues that the complexity measure should not be dependent on the type of characteristic (i.e., STB, NTB, or LTB). This paper makes an effort to eliminate this disparity among the three relationships.

Joseph (2004) addressed QLF for nonnegative variables. That is, Joseph derives a new set of loss functions for nonnegative variables using Taguchi's definition of quality as a basis. The proposed loss functions assume that the loss is additive and employs STB, nominal-the-better (NTB), and LTB. The new loss function is compared with the quadratic loss function and is shown to be comparable since the quadratic loss function is meant for unrestricted variables. Joseph also proposed a multivariate extension of the QLF.

Similarly, El-Haik (2005) also linked design complexity to SN ratio for the three types of quality engineering characteristics (called functional requirements, $F R$, in axiomatic design) because both complexity and SN ratio are functions of variability. The relationships between axiomatic complexity and SN ratio for STB, NTB, and LTB characteristics are also different. This is also attributed to the use of a reciprocal transformation for the LTB type of characteristic. The mathematical relationships between complexity and SN ratios should not be dependent on the type of characteristic (Sharma et al, 2009). In this paper an attempt has been made to bring about similarity among the three cases by introducing linear transformation for the LTB case as used for the other two cases. The methodology proposed leads to consistent and comparable results because it obviates the need to assume the target value as infinity for LTB characteristics. This consistency is not possible in the approach given in El-Haik (2005), which is based on an infinite target for LTB. In the proposed method, a common method for all three cases (i.e., STB, NTB, and LTB) can be used for the relationship of 1) complexity and quality loss and 2) complexity and SN ratio. In addition, the relationships become equivalent for STB and LTB cases at a target-mean ratio of 2. By utilizing the unified loss function an attempt has been made to streamline the mathematical aspect as well as the practical aspect of the relationship between complexity and the quality loss function as well as between complexity and SN ratio. In the next section the unified quality loss function is briefly revisited. Then the SN ratio for LTB characteristics based on the unified loss function is presented.

\section{Unified Quality Loss Function}

Mean squared deviation (MSD) is used in the formulation of the quality loss function for STB, NTB, and LTB characteristics (Maghsoodloo, 1991). The term $\left(y_{i}-m\right)^{2}$ is the mean squared deviation, for a group of $n$ products given the performance readings

of $y_{i}=y_{1}, y_{2}, y_{3}, \mathrm{~K}, y_{n}$ and a target of $m$. In Sharma et al (2007), a unified formula, shown in Equation 1, was proposed for the three types of characteristics, namely STB, NTB, and LTB.

$$
M S D=\frac{1}{n} \sum_{i=1}^{n}\left(y_{i}-m\right)^{2}=\sigma^{2}+(\bar{y}-m)^{2}=\sigma^{2}+\bar{y}^{2}(1-\alpha)^{2}
$$

Where $\bar{y}$ is the mean of the present performance and $\alpha=m / \bar{y}$ is the target-mean ratio. When $\alpha=0$, Equation 1 represents the STB case. When $\alpha \approx 1$, this equation represents the NTB case. Finally, when $\alpha=2$ (or any other finite value), Equation 1 represents the LTB case. The target, $m$, may be chosen such that all $y_{i}>m$. The chosen target may be kept constant for subsequent improvement processes for the same system. LTB can be transformed to STB using $y_{i}-m$ or $m-y_{i}$ because MSD will be the same in either case. It is recommended to use $m-y_{i}$ according to Equation 2 in order to keep the converted STB (from LTB) always positive. 


$$
M S D=\frac{1}{n} \sum_{i=1}^{n}\left(m-y_{i}\right)^{2}=\sigma^{2}+(m-\bar{y})^{2}
$$

There are many LTB performance parameters which have no obvious ideal performance limit. This is the basis for the assumption of an infinite target and the use of the reciprocal transformation for LTB in Taguchi's loss function. To facilitate the use of linear transformation in place of reciprocal transformation, the target can be determined by selecting a target-mean ratio for LTB. In the case of LTB, $\alpha$ must be significantly greater than 1 but need not be a large number or infinity. It has been shown by mathematical derivation and a case study that at $\alpha=2$ the LTB case is equivalent to the STB case. The only difference is that the target is not zero but is placed equidistant from the mean on the other side. Therefore, it was recommended to set the target at double the present mean performance for most cases (Sharma et al, 2007). The target is not assumed to be infinity though the case is LTB. Therefore, Equation 1 can be used interchangeably to encompass all three cases of quality loss (STB, NTB, and LTB).

In the next section an overview of the current SN ratio for LTB is given and then a new SN ratio based on a finite target is proposed. An example of a welding process is given to demonstrate the use of the proposed SN ratio. Next, the existing complexity formulations for STB, NTB, and LTB are discussed and a new complexity formulation for LTB is proposed based on a finite target. Then, the existing relationships between the SN ratio and complexity for LTB cases are discussed and a new relationship between SN ratios and complexity is proposed based on a finite target. An example is given to show that the proposed relationship between the SN ratios and complexity for LTB gives comparable and consistent results.

\section{Signal-to-Noise Ratio for LTB with a Finite Target}

The SN ratio functions as a single measure of robustness and a gain in the SN ratio reflects an improvement in robustness (Taguchi, 2004). This ratio must be related to lower cost, reduced time to market, and improved quality. In general, the SN ratio for quality characteristics is based on mean squared deviation and can be calculated as given in Equation 3 (Taguchi, 2004; Fowlkes and Creveling, 1995).

$$
S / N=-10 \log _{10}(M S D)
$$

Taguchi (2004) states that "The larger-the-better characteristic should be nonnegative, and its most desirable value is infinity." Joglekar (2003) argued "Characteristics such as bond strength also do not have negative values but larger values are preferred. The target value is infinity. Such characteristics are called larger-the-better characteristics.” Yang and El-Haik (2008) have discussed "For some quality characteristics, such as welding bond strength, the ideal target value is infinity. These are larger-thebetter (i.e., "the larger, the better" or "larger is better") quality characteristics. Performance levels will progressively worsen if $y$ decreases; the worst possible value is zero." For the worst possible case at zero, Taguchi's quality loss is infinite because the reciprocal of zero is infinite. The concept of a finite target for LTB is used as the basis for the development of the SN ratio, design complexity, and the relationship between them. For LTB characteristics, Taguchi's signal-to-noise ratio also assumes a target of infinity, which is given in Equation 4 (Fowlkes 1995; Taguchi, 1999; Taguchi, 2004).

$$
S / N=-10 \log _{10}(M S D)=-10 \log _{10}\left(\frac{1}{n} \sum_{i=1}^{n} \frac{1}{y_{i}^{2}}\right) \approx \frac{1}{\bar{y}^{2}}\left(1+\frac{3 \sigma^{2}}{\bar{y}^{2}}\right)
$$

The assumption of an unrealistic infinite target for LTB induces several issues as discussed in Sharma et al (2007). The quality loss estimates for STB and LTB are not comparable. The MSD for LTB in terms of variance and mean of performance is approximate only and employs variance and mean of the performance, not bias as shown in Equation 4. The bias is infinity in every case before improvement as well as after improvement (Sharma et al, 2007). Therefore, the MSD or SN ratio cannot depend on bias.

If the maximum possible target (e.g., 100\% efficiency) is achieved, Taguchi's loss function delivers a small quality loss, though not zero as expected. Also, the loss is infinite when the observed value for the LTB characteristic is zero. Taguchi's quality loss function based on the reciprocal transformation does not satisfy both the boundary conditions, i.e., extremely good and extremely poor performance. In Sharma et al (2007) it was demonstrated that the quality loss is zero if the maximum possible target is achieved when the unified quality loss function is employed. Moreover, the unified loss function gives a finite loss when the LTB characteristic approaches zero. Therefore, the unified loss function produces realistic results at all increments including the ideal boundary conditions. Therefore, it is proposed to employ the unified loss function that uses a finite target and linear transformation for LTB in place of Taguchi's loss function which uses an infinite target and reciprocal transformation.

Sharma et al (2008) proposed the concept of the complementary property. An STB characteristic complementing a given LTB characteristic may be called a complementary characteristic. For example, inefficiency (STB) is a complementary characteristic of efficiency (LTB), and percent defective (STB) is a complementary characteristic of percent non-defective (LTB). It was suggested that whether a given LTB characteristic or its STB complementary characteristic is considered for the same process or system the $M S D$ and quality loss must be equal. In other words, the quality loss must satisfy the complementary property. In general, if the target is infinity, then the MSD or quality loss for LTB and that for its complementary characteristic are unequal and the complementary property is not satisfied. The unified loss function satisfies the complementary property without a loss of generality. 
It is proposed that a finite target, $m$, be considered for LTB. Therefore, the proposed SN ratio using linear transformation as suggested in Sharma et al (2007) is employed in place of reciprocal transformation is given as shown in Equation 5.

$$
M S D=\frac{\sum\left(y_{i}-m\right)^{2}}{n}=\sigma^{2}+(\bar{y}-m)^{2}
$$

Therefore,

$$
S / N=-10 \log _{10}\{M S D\}=-10 \log _{10}\left\{\sigma^{2}+(\bar{y}-m)^{2}\right\}=-10 \log _{10}\left\{\sigma^{2}+\bar{y}^{2}(1-\alpha)^{2}\right\}
$$

In the proposed Equation 6, the MSD for LTB has two components including variance and bias. This is the same as the MSDs for STB and NTB. Improved performance with respect to a higher target reduces bias. This reduction in bias is neither obvious nor observable in Taguchi's MSD for LTB. Reduction in either variance or bias or both will reduce the MSD and increase the proposed SN ratio.

In Taguchi's method, LTB must be converted to STB using reciprocal transformation. When reciprocal transformation is used to convert LTB into STB several issues arise. First, the reciprocals of performance values greater than one will be between zero and one. This compression or change of scale is unnecessary and can be eliminated. By avoiding reciprocal transformation and using simple linear transformation the full and actual range of performance can be utilized in calculations. Taguchi's, as well as the unified loss function, are independent of any particular distribution of performance. The performance values are expected to follow some distribution with the most common being the normal distribution. When a variable follows a normal distribution the reciprocal of the variable follows an inverse normal distribution. However, according to Robert (1991), the mean and variance of the inverse normal distribution are undefined. It is, therefore, evident that the reciprocal of a performance value does not retain the information of the response because of the nature of the transformation. In general, the distribution of a reciprocal of response changes from that of the response itself.

Second, the unit of response changes to some other unit that in many cases is not really useful or comprehensible. For example, pressure in lbs/in ${ }^{2}$ will change to in ${ }^{2} / \mathrm{lb}$, temperature in ${ }^{0} \mathrm{C}$ will change to ${ }^{0} \mathrm{C}^{-1}$, and tool life in hrs will change to hrs ${ }^{-1}$. In comparison, such a change of unit is not required for STB or NTB. Third, the calculations no longer remain simple and exact because approximation is achieved using Taylor series expansion of the reciprocal as shown in Equation 4. Next, the results are no longer comparable with the STB characteristic (Sharma et al, 2007). Finally, if one were to combine different characteristics in the case of multivariate quality loss when LTB needs to be considered along with STB or NTB, they would not be comparable. In that situation, it would make more sense to use the covariance between STB (or NTB), $y_{i}$, and LTB, $y_{j}$, in comparison to using the covariance between STB (or NTB), $y_{i}$, and LTB, $1 / y_{j}$, in computations.

The linear transformation does not alter the variance of the original data, whereas reciprocal transformation completely alters the variance. Using linear transformation with a target at 2 times the mean renders an important advantage that the mean of the data is also unchanged along with the variance. Reciprocal transformation alters the mean as well as the variance of the original data. In this way, the linearly transformed data is used as if the data was not transformed in terms of the mean and variance. Suppose the same data set that pertains to the STB characteristic, also pertains to the LTB characteristic. The SN ratios computed using present method will be altogether different merely because the characteristic types are different. With the proposed method equivalent ratios for STB and LTB are obtainable.

Therefore, it is proposed that Equation 6 be used interchangeably to encompass the three cases. At $\alpha=0$, Equation 6 provides the SN ratio for the STB case. When $\alpha \approx 1$, the equation gives the SN ratio for the NTB case. Finally, when $\alpha=2$ (or any other finite value), Equation 6 gives the SN ratio for the LTB case. As discussed in the previous unified quality loss function section, it is recommended to set the target at double the present mean performance or $\alpha$ is set equal to 2 . The following example demonstrates how the proposed SN ratio for LTB can be used to measure improvement.

\section{Example 1: Welding Process}

This example is taken from Fowlkes (1995) in which welding is performed using four welding machines and the interchanging of three operators is treated as a noise factor. The LTB quality characteristic in this problem is welding strength measured in pound per square inch (psi).

Table 1 shows the calculations of mean performance, MSD, quality loss in dollars, and SN ratio using the reciprocal transformation in Taguchi's loss function for the four welding machines in the presence of noise, i.e., changing operators from shift to shift. The last column gives the ranking of machines on the basis of SN ratio calculated using Taguchi's loss function as given in Equation 4. Also in this table, an overall mean performance $(\bar{y}=6188.33)$ is shown which is used to set the target in Table 2. The SN ratios using reciprocals for non-negative quality characteristics greater than one should be positive. As shown in Table 2, these values are positive for all four machines as the performance values for all the machines are greater than one.

Table 2 also shows the values of performance deviations from the mean $\left(m-y_{i}\right), M S D$, and SN ratio using the unified loss function, as given in Equation 6, in the presence of the same noise. The last column in Table 2 provides the ranking of machines 
on the basis of SN ratio using the unified loss function. The ranking of machines using the new LTB quality loss function is the same as that obtained using Taguchi's loss function. The MSD function proposed is monotonous for $y_{i}<m$. Therefore, the unified quality loss function can be used to provide consistent results for the SN ratio. The SN ratios using linear transformation for non-negative quality characteristics greater than one should be negative. As shown in Table 2, the SN ratios are negative as expected for all four machines as the performance values for all the machines are greater than one.

Table 1. Machine ranking on the basis of SN ratio calculated using Taguchi's quality loss

\begin{tabular}{||l|l|l|l|l|l|l|l||}
\hline \hline $\begin{array}{l}\text { Machine } \\
\text { No. }\end{array}$ & Noise 1 & Noise 2 & Noise 3 & Mean & MSD & $\begin{array}{l}\text { SN } \\
\text { ratio }\end{array}$ & Machine rank \\
\hline 1 & 9340 & 3030 & 7830 & 6733.333 & $4.56 \times 10^{-8}$ & 73.41 & Third best \\
\hline 2 & 2980 & 2450 & 3100 & 2843.333 & $1.28 \times 10^{-7}$ & 68.94 & Worst machine \\
\hline 3 & 7890 & 9100 & 8340 & 8443.333 & $1.42 \times 10^{-8}$ & 78.49 & Best machine \\
\hline 4 & 6550 & 6700 & 6950 & 6733.333 & $2.21 \times 10^{-8}$ & 76.56 & Second best \\
\hline \multicolumn{7}{|l|}{ Mean performance of four machines $=(6733.333+2843.333+8443.333+6733.333) / 4=6188.33$} \\
\hline
\end{tabular}

Table 2. Machine ranking on the basis of SN ratio calculated using the unified loss function

\begin{tabular}{|c|c|c|c|l|l|l|l|l|l||}
\hline $\begin{array}{c}\text { Machine } \\
\text { No. }\end{array}$ & $\begin{array}{c}\text { Noise } \\
\mathbf{1}\end{array}$ & $\begin{array}{c}\text { Noise } \\
\mathbf{2}\end{array}$ & $\begin{array}{c}\text { Noise } \\
\mathbf{3}\end{array}$ & $\mathbf{m}-\mathbf{y}_{\mathbf{1}}$ & $\mathbf{m}-\mathbf{y}_{\mathbf{2}}$ & $\mathbf{m}-\mathbf{y}_{\mathbf{3}}$ & $\mathbf{M S D}$ & $\begin{array}{l}\text { SN } \\
\text { ratio }\end{array}$ & Machine rank \\
\hline 1 & 9340 & 3030 & 7830 & 3036.67 & 9346.67 & 4546.67 & 39084567 & -75.92 & Third best \\
\hline 2 & 2980 & 2450 & 3100 & 9396.67 & 9926.67 & 9276.67 & 90964200 & -79.59 & Worst machine \\
\hline 3 & 7890 & 9100 & 8340 & 4486.67 & 3276.67 & 4036.67 & 15720467 & -71.96 & Best machine \\
\hline 4 & 6550 & 6700 & 6950 & 5826.67 & 5676.67 & 5426.67 & 31874433 & -75.03 & Second best \\
\hline
\end{tabular}

In the example of the welding strength one might say 6000 is better than 3000,12000 is better than 6000,24000 is better than 12000 , and this goes on at infinitum. As previously discussed, the target, $m$, should preferably be set at 2 times the mean provided $m$ is greater than all $y_{i}$. This ensures that there would not arise any need to consider a situation such as 13000 is better than 12376 psi in which $\left(13000=y_{i}\right)>(12376=m)$. This violates the given condition " $m>$ all $y_{i}$ " and, therefore, has no place in the loss function proposed. Suppose there were a $\left(y_{i}=13000\right)$, then $m$ would be greater than 13000 with a target-mean ratio more than 2 but finite. This is expected to be a possible rare case. For example, it is unlikely that a performance weld strength reading of $y_{i}=$ 13000 is part of the data set given in the example that has a mean of 6188.33. However, when it is observed, then the condition that the target must be greater than all $y_{i}$ must be satisfied when setting the target. When such a situation arises a target-mean ratio higher than 2 may be used. The next section discusses the formulations of complexity for STB and NTB, using a finite target, LTB using an infinite target, and the use of the unified loss function. The relationships between the SN ratios and complexity are discussed in a later section.

\section{Complexity and Quality Loss - LTB with Finite Target}

Complexity is defined as a measure of uncertainty in achieving the specified functional requirements (FR) (Suh, 2001). Therefore, complexity is related to information content, which is defined as a logarithmic function of the probability of achieving functional requirements, which in turn is information content. The design process is described in terms of the mapping between domains in axiomatic design (Suh, 1999). Functional requirements in the functional domain describe the design goals for a product, e.g., systems and software. In the design stage, the functional requirements in the functional domain are mapped to the design parameters (DP) in the physical domain (Suh 1999). The probability of success means the probability of satisfying a given FR in the process domain. The process domain allocates the processes required to achieve the design parameters and to realize products. A design parameter is distributed over the system range. Therefore, the process domain can be viewed as the system range. The design range is defined as the acceptable tolerance range for a functional requirement. Therefore, the probability of success would be the area under the curve of the probability density function (pdf) for the system range bounded by the design range (Suh, 1999). The design range is specified using lower and upper specification limits, i.e., LSL $\left(d r^{l}\right)$ and USL $\left(d r^{u}\right)$, as 
shown in Figure 1. The system pdf has an influence on the system range ( $s r^{l}$ to $s r^{u}$, Figure 1) and the system range provides bounds on the system pdf. However, the system range may be different from the design range. For a quality characteristic, $y$, in robust design the corresponding term in axiomatic design is the FR. The common range ( $s r^{l}$ to $d r^{u}$, Figure 1) is the portion of the system range overlapped by the design range. The common area under the system pdf and design range, in turn, gives the probability of achieving a specified FR (El-Haik, 2005).

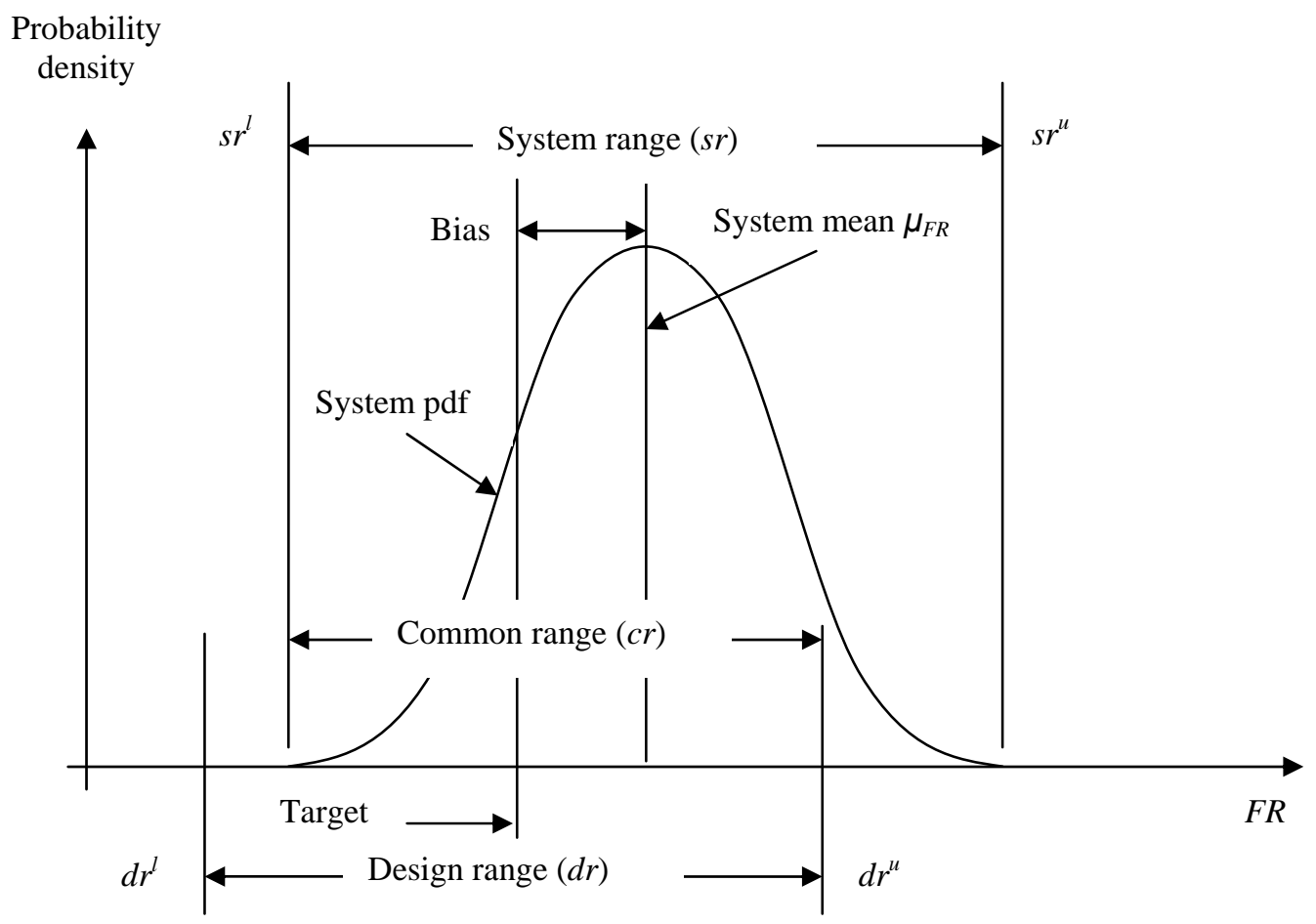

Figure 1. Design range, system range, and common range

The information axiom in axiomatic design is to "minimize the information content of the design" (Suh, 2001; Lee, 2003). The design or manufacturing of a given component requiring minimum information content gives the maximum probability of achieving success. According to El-Haik (2005), Shannon entropy is restricted to discrete random variables. Differential entropy, employing the concept of Boltzmann entropy, is used to compute the complexity for functional requirements that follow continuous probability distributions. The complexity due to variability for the interval $\left[\mu_{\mathrm{FR}}-\Delta \mathrm{FR}, \mu_{\mathrm{FR}}+\Delta \mathrm{FR}\right]$ is given as in Equation 7 (El-Haik, 2005):

$$
h(f)=-\int_{\mu_{F R}-\triangle F R}^{\mu_{F R}+\Delta F R} f(F R) \ln f(F R) d F R
$$

Complexity for a given functional requirement that follows a normal system range (a normal source of information) i.e., $F R \sim \operatorname{Nor}\left(\mu_{F R}, \sigma_{F R}^{2}\right)$, using approximation is given as in Equation 8 (El-Haik, 2005).

$$
h(f)=\ln \sqrt{2 \pi e \sigma_{F R}^{2}}
$$

El-Haik’s work bridges the gap between robust design and axiomatic design by providing mathematical relationships between quality loss and complexity. Complexity reduction is shown to be related to reduction in quality loss. Further, the relationships between complexity and the quality loss function concerning STB, NTB, and LTB are given in El-Haik (2005) as follows. For $\mathrm{NTB}$, if the target is denoted as $T_{F R}$, and $k$ is a proportionality constant, then the quality loss is given as in Equation 9.

The expected loss is shown in Equation 10.

$$
L\left(F R, T_{F R}\right)=k\left(F R-T_{F R}\right)^{2}
$$

$$
\begin{aligned}
& E\left[L\left(F R, T_{F R}\right)\right]=k\left[\left(\mu_{F R}-T_{F R}\right)^{2}+\sigma_{F R}^{2}\right] \\
\Leftrightarrow & \sigma_{F R}^{2}=\frac{E\left[L\left(F R, T_{F R}\right)\right]}{k}-\left(\mu_{F R}-T_{F R}\right)^{2}
\end{aligned}
$$


Replacing $\sigma_{F R}^{2}$ in Equation 8 gives the complexity as shown in Equation 11 (El-Haik, 2005):

$$
h(f)=\ln \sqrt{2 \pi e\left(\frac{E\left[L\left(F R, T_{F R}\right)\right]}{k}-\left(\mu_{F R}-T_{F R}\right)^{2}\right)}
$$

where,

$$
E\left[L\left(F R, T_{F R}\right)\right]>k\left(\mu_{F R}-T_{F R}\right)^{2}
$$

Equation 11 can also represent the STB case when $T_{F R}=0$. However, for LTB, where the target is infinity and the $F R$ is bounded by $F R_{l}$, the lower specification limit, the loss is shown in Equation 12.

$$
\begin{aligned}
& L\left(F R, T_{F R}\right)=\frac{k}{F R^{2}} \\
& F R \geq F R_{l}
\end{aligned}
$$

If $\mu_{F R}$ is the mean $F R$ of the system range, then by Taylor series expansion around $F R=\mu_{F R}$ Equation 13 is determined by neglecting higher-order terms greater than two (El-Haik, 2005).

$$
L\left(F R, T_{F R}\right)=\left.k\left(\frac{1}{\mu_{F R}^{2}}+\frac{3}{\mu_{F R}^{4}} \sigma_{F R}^{2}\right)\right|_{F R=\mu_{F R}}
$$

For a normally distributed $F R$, an approximate solution for complexity is provided as shown in Equation 14 (El-Haik, 2005).

$$
h(f)=\ln \sqrt{2 \pi e\left(\frac{2 \pi \mu_{F R}^{4} E\left[L\left(F R, T_{F R}\right)\right]}{3 k}-\frac{2 \pi \mu_{F R}^{2}}{3}\right)}
$$

where,

$$
E\left[L\left(F R, T_{F R}\right)\right]>\frac{k}{\mu_{F R}^{2}}
$$

Because STB and NTB consider a finite target whereas LTB considers an infinite target, the formula for complexity due to variability for LTB, (Equation 14) is different from that for NTB and STB cases (Equation 11). Also, because complexity as defined by Equation 8 only depends on the variance of performance $\sigma_{F R}^{2}$ and the terms inside the round brackets in Equations 11 and 14 are simply a replacement for $\sigma_{F R}^{2}$, the complexity due to variability for all three types of characteristics will be equal for the same standard deviation of performance. To achieve similarity among complexity for the three cases it is proposed that a finite target be considered for LTB. When a finite target in terms of the target-mean ratio is considered for LTB then the proposed solution is found using Equation 11 and $\alpha=T_{F R} / F R$ as follows in Equation 15:

$$
h(f)=\ln \sqrt{2 \pi e\left(\frac{E\left[L\left(F R, T_{F R}\right)\right]}{k}-\mu_{F R}^{2}(1-\alpha)^{2}\right)}
$$

where,

$$
E\left[L\left(F R, T_{F R}\right)\right]>k \mu_{F R}^{2}(1-\alpha)^{2}
$$

For a particular case when $\alpha=2$,

$$
h(f)=\ln \sqrt{2 \pi e\left(\frac{E\left[L\left(F R, T_{F R}\right)\right]}{k}-\mu_{F R}^{2}\right)}
$$

It is proposed that Equation 15 be used interchangeably to cover three cases. At $\alpha=0$, Equation 15 gives the complexity for the STB case, when $\alpha \approx 1$ this equation gives the complexity for NTB case, and when $\alpha=2$ (or any other finite value) Equation 16 gives the complexity for the LTB case.

The complexity for the LTB case at $\alpha=2$ is equivalent to that for the STB case when $\alpha=0$. Therefore, comparable results for complexity can be obtained using the unified loss function. In the next section, the relationship between SN ratio and complexity is discussed as well as a simplification approach using the unified quality loss function in which LTB has a finite target. 


\section{SN Ratio and Complexity Relationship}

Design complexity and SN ratio are both functions of variation. However, both are related with variation in opposing fashion. As variation increases complexity also increases but SN ratio decreases. The ideal system has a high SN ratio and low complexity. The SN ratio is a function of bias (or mean) of performance and variance. El-Haik (2005) connected robust design with axiomatic design through mathematical relationships between complexity and $\mathrm{SN}$ ratios for the three types of quality characteristics. According to El-Haik (2005), Equation 17 relates the SN ratio and complexity for LTB.

$$
S N=-10 \log _{10}\left(\frac{3\left[e^{h(f)}\right]^{2}+2 \pi e \mu_{F R}^{2}}{2 \pi e \mu_{F R}^{4}}\right)
$$

According to El-Haik (2005), Equation 18 relates the SN ratio for STB and complexity:

$$
h(f)=\ln \sqrt{2 \pi e\left(10^{-\frac{S N}{10}}-\mu_{F R}^{2}\right)}
$$

The relationships between SN ratio and complexity for LTB (Equation 17) and STB (Equation 18) are neither equivalent nor similar. Therefore, the results would generally be different. This inconsistency can be eliminated using linear transformation in the unified loss function in place of reciprocal transformation used by Taguchi for an infinite target. With a finite target using the unified loss function, as proposed earlier the MSD and SN ratio for LTB can be related as in Equation 19 as follows (from Equation 6):

$$
10^{-\frac{S N}{10}}=\frac{1}{N} \sum_{i=1}^{n}\left(F R_{i}-T_{F R}\right)^{2}=\left(\mu_{F R}-T_{F R}\right)^{2}+\sigma_{F R}^{2}=\frac{E\left[L\left(F R, T_{F R}\right)\right]}{k}
$$

Therefore, it is proposed that the SN ratio and complexity for LTB characteristics be related in a different way as follows in Equation 20 (from Equations 15 and 19):

$$
S N=-10 \log _{10}\left(\frac{\left[e^{h(f)}\right]^{2}}{2 \pi e}+\mu_{F R}^{2}(1-\alpha)^{2}\right)
$$

When $\alpha=2$,

$$
\begin{aligned}
& h(f)=\ln \sqrt{2 \pi e\left(10^{-\frac{S N}{10}}-\mu_{F R}^{2}\right)} \\
& S N=-10 \log _{10}\left(\frac{\left[e^{h(f)}\right]^{2}}{2 \pi e^{2}}+\mu_{F R}^{2}\right)
\end{aligned}
$$

Equation 20 can be used interchangeably to describe the three cases. At $\alpha=0$, Equation 20 gives the relationship between the complexity and SN ratio for the STB case. When $\alpha \approx 1$ this equation gives the relationship between complexity and SN ratio for the NTB case. Finally, when $\alpha=2$ (or any other finite value) Equation 20 gives the relationship between complexity and SN ratio for the LTB case. For LTB at $\alpha=2$, the relationship between the SN ratio and the complexity measure becomes equivalent and similar to that for STB. This equivalence is not obvious with Taguchi's method when one compares Equations 20 and 17.

Although the current relationships between complexity and quality loss are different for LTB and NTB (or STB) the complexity measures are the same for all three cases. This is because the complexity equations take into account standard deviation only. The $\mathrm{SN}$ ratio based on complexity will generally be different for different types of characteristics when the reciprocal transformation is used for calculating the SN ratio. On the other hand, the SN ratio based on the unified loss function provides consistent complexity among the three cases. The following section provides an example to demonstrate this consistency.

\section{Example 2: SN Ratio and Complexity - Spring Rate}

This example is taken from Fowlkes (1995) and El-Haik (2005). The sample consists of two sets of springs manufactured by two machines, new and old. Their spring rates are given in Table 3. The spring rate is an NTB characteristic with a target value of $0.5 \mathrm{oz} . / \mathrm{in}$. Spring rate complexity and signal-to-noise ratio based on complexity for each machine is calculated. Tables 3 and 4 give the calculated values of complexity using Equations 11 and 14 for the three cases as if the data set pertained to NTB, STB, 
and LTB characteristics, respectively. The complexity measures are the same for all three cases because the complexity equations take into account the standard deviation only.

Table 3. Complexity of the Spring Rate- NTB

\begin{tabular}{|c|c|c|c|c|c|c|c|c|c|c|c|c|c|c|}
\hline \multirow{2}{*}{$\begin{array}{l}\stackrel{\Xi}{\Xi} \\
\text { : } \\
\sum \\
\Sigma\end{array}$} & \multirow{2}{*}{\multicolumn{8}{|c|}{ Spring rate data }} & \multirow{2}{*}{ 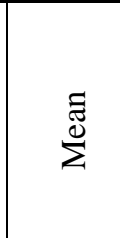 } & \multirow{2}{*}{ 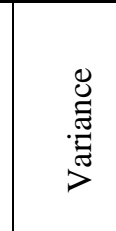 } & \multicolumn{4}{|c|}{ NTB } \\
\hline & & & & & & & & & & & $\begin{array}{l}\varepsilon \\
\mathbb{\Phi}\end{array}$ & & क & $\stackrel{\vec{x}}{\stackrel{\vec{x}}{a}}$ \\
\hline New & 0.37 & 0.41 & 0.37 & 0.43 & 0.39 & 0.35 & 0.4 & 0.36 & 0.385 & 0.0007 & 0.5 & 0.0132 & 0.014 & -2.184 \\
\hline Old & 0.55 & 0.67 & 0.7 & 0.54 & 0.41 & 0.32 & 0.46 & 0.66 & 0.5388 & 0.0184 & 0.5 & 0.0015 & 0.0199 & -0.579 \\
\hline
\end{tabular}

However, this situation does not hold if the SN ratios are calculated using complexity. The SN ratios computed using Equation 17 with the assumption of the target at infinity for LTB (-8.3556 and -0.5792, new and old machine, respectively) are not the same as for STB (8.26907 and 5.10554 new and old machine, respectively) as shown in Table 4. For STB and LTB, the SN ratios have different values. This inconsistency in the results is generally inevitable if the target is infinity. NTB is not considered here as comparison since NTB is not warranted.

Table 4. SN Ratios Based on Complexities of the Spring Rate-STB and LTB

\begin{tabular}{|c|c|c|c|c|c|c|c|c|}
\hline \multirow[b]{3}{*}{$\operatorname{Bias}^{2}$} & \multicolumn{3}{|c|}{ STB } & \multicolumn{5}{|c|}{ LTB } \\
\hline & \multirow[b]{2}{*}{$M S D$} & \multirow[b]{2}{*}{ 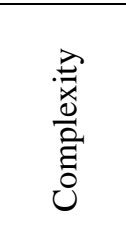 } & \multirow{2}{*}{ 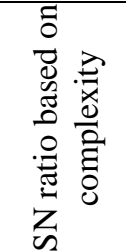 } & \multirow[b]{2}{*}{ 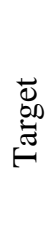 } & \multirow[b]{2}{*}{ 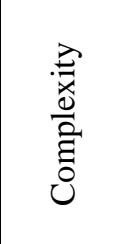 } & \multicolumn{3}{|c|}{ SN ratio based on complexity } \\
\hline & & & & & & $\begin{array}{c}\text { Existing } \\
\text { method, } \\
\text { infinite } \\
\text { target }\end{array}$ & $\begin{array}{c}\text { Finite } \\
\text { target at } \\
\text { alpha }=1.5\end{array}$ & $\begin{array}{c}\text { Finite } \\
\text { target } \\
\text { alpha=2 }\end{array}$ \\
\hline 0.14823 & 0.14897 & -2.1836 & 8.26907 & $\infty$ & -2.1836 & -8.3556 & 14.2252 & 8.26907 \\
\hline 0.29025 & 0.30864 & -0.5792 & 5.10554 & $\infty$ & -0.5792 & -6.1278 & 10.4121 & 5.10554 \\
\hline
\end{tabular}

The proposed Equation 20 was used to calculate the SN ratio for LTB based on complexity at 1.5 and 2 alpha levels, respectively. The SN ratios calculated with the assumption of a finite target at the target-mean ratio (i.e., alpha level) of 2 are the same for LTB (8.26907 and 5.10554 new and old machine, respectively) as for STB (8.26907 and 5.10554 new and old machine, respectively). Therefore, the SN ratio and complexity for LTB characteristics can be related as proposed in Equation 20 to obtain consistent and comparable results.

\section{Conclusions}

This paper considers Taguchi's quality loss for calculating the SN ratio for LTB in which reciprocal transformation is used to convert LTB into STB. Reciprocal transformation makes it difficult to deal with and alters some results. Examples include the SN ratio based on mean squared deviation $(M S D)$ and the SN ratio based on complexity. The unified method assumes some finite target in the case of LTB. The implication of a finite target on SN ratio, the relationship between the quality loss function and complexity, and the relationship between the SN ratio and complexity has also been discussed in this paper. The complexity formulation for LTB with an infinite target is not similar to that for STB and LTB characteristics. They need to be similar because the type of characteristics should not determine the complexity formulation. Therefore, complexity due to variability for LTB with a finite target was derived and shown to have a comparable complexity measure with the unified quality loss function. The effect of a finite target for LTB on the mathematical relationship between the signal-to-noise ratio and axiomatic measures was also studied. This relationship between signal-to-noise ratio and complexity, an axiomatic measure, becomes equivalent to the STB case. A simple linear transformation that assumed a finite target was shown to provide comparable SN ratios based on the MSD and SN ratio based on complexity for LTB characteristics. Two examples were also discussed to demonstrate the proposed methodology.

\section{Nomenclature \\ DP Design parameter \\ FR Functional requirement \\ LTB Larger-the-better}


NTB Nominal-the best

SN Signal-to-noise ratio

STB Smaller-the-better

\section{References}

El-Haik, B.S., (2005), Axiomatic Quality - Integrating Axiomatic Design with Six-Sigma, Reliability, and Quality Engineering, Wiley-Interscience Publication, John Wiley \& Sons, Inc., Hoboken, NJ.

El-Haik, B.S., (2006), “Axiomatic Quality: A Framework for Axiomatic Design \& Robust Integration, Robust Engineering, ASI’s $20^{\text {th }}$ Annual Symposium, Livonia, MI.

Fowlkes, W.Y., and Creveling, C.M., (1995), Engineering Methods for Robust Product Design, Addison-Wesley Publishing Company, Reading, MA.

Joglekar, A.M., (2003), Statistical Methods for Six Sigma, In R \& D and Manufacturing, Wiley-Interscience, John Wiley \& Sons, Inc., Hoboken, NJ.

Joseph V.R. (2004), Quality loss functions for nonnegative variables and their applications; Journal of Quality Technology 36; 129-138.

Kar, A.K., (2000), Linking axiomatic Design and Taguchi Methods via Information Content in Design, Proceedings of ICAD2000, First International Conference on Axiomatic Design, Cambridge, MA, ICDA033, pp 219-224.

Lee, T., (2003), Complexity Theory in Axiomatic Design, PhD Dissertation, Massachusetts Institute of Technology.

Maghsoodloo S. (1991), The exact relationship of Taguchi's signal-to noise ratio to his quality loss function; Journal of Quality Technology 22(1); 57-67.

Robert, C., (1991), Generalized Inverse Normal Distributions, Statistics \& Probability Letters, 11, January, 1991, pp. 37-41.

Sharma, N.K., Cudney, E.A., Ragsdell, K.M., and Kioumars, P., (2007) "Quality Loss Function - A Common Methodology for Three Cases,” Journal of Industrial and Systems Engineering, Vol. 1, No. 3, pp. 218-234.

Sharma, N.K., Cudney, E.A., Drain, D., and Ragsdell, K.M., (2008), Implications of Quality Loss Function in Unified Methodology - LTB Case with Target, SAE International Journal of Materials and Manufacturing, Vol. 1, No. 1, pp 768-777.

Sharma, N.K., Cudney, E.A., and Sharma, S., (2009), Design Complexity and its Relationship with SN Ratio - Larger-the-better Characteristics with Finite Target, SAE World Congress, Detroit, Michigan, Paper No. 2009-01-0797.

Suh, N.P., (1999), A Theory of Complexity, Periodicity and Design Axioms, Research in Engineering Design, 11. pp. 116-131.

Suh, N.P., (2001), Axiomatic Design - Advances and Applications, Oxford University Press, New York, NY.

Taguchi, G., Chowdhury, S., and Taguchi, S., (1999), Robust Engineering, McGraw Hill, New York, NY.

Taguchi, G., Chowdhury, S., and Wu, Y., (2004), TAGUCHI'S Quality Engineering Handbook, John Wiley \& Sons, Inc., Hoboken, NJ.

Yang, K., and El-Haik, B., (2008), Design for Six Sigma: A Roadmap for Product Development, McGraw Hill, New York, NY.

\section{Biographical notes}

Dr. N.K. Sharma has a doctorate in Engineering Management from the Missouri University of Science \& Technology. He has a bachelors in Mechanical Engineering and masters in Engineering Management and Financial Management from India. He has over 10 years of experience of overhaul of coaches of Indian Railway and nearly 6 years of experience of maintenance of plant and machinery. This work involved middle level management of activities for achieving corporate objectives of productivity targets. He is a Certified Lead Assessor for ISO-9000. He is familiar with production activities e.g., planning and scheduling, quality engineering. He has also worked in the areas of design of components and mechanical systems. His research interests include engineering design, robust engineering, and warranty cost prediction and reduction. His email address is nkscnc@mst.edu.

Dr. E.A. Cudney is an Assistant Professor at Missouri University of Science and Technology. She received her B.S. in Industrial Engineering from North Carolina State University, Master of Engineering in Mechanical Engineering and Master of Business Administration from the University of Hartford, and her doctorate in Engineering Management from the University of Missouri - Rolla. In 2010, Dr. Cudney was inducted into the ASQ International Academy for Quality. She received the 2008 ASQ A.V. Feigenbaum Medal and the 2006 SME Outstanding Young Manufacturing Engineering Award. She is an ASQ Certified Quality Engineer, Manager of Quality/Operational Excellence, and Certified Six Sigma Black Belt. She is a member of the ASEM, ASME, ASQ, IIE, SAE, and the Japan Quality Engineering Society (JQES).

Received January 2011

Accepted June 2011

Final acceptance in revised form June 2011 